\title{
Experimental Investigation of the Characteristics of the Information Sensing Module Based on the Optical Tunneling Effect of the Angular Velocity Transducer by Using a Piezoelectric Module
}

\author{
V. I. Busurin, A. V. Kazaryan, Yin Naing Win, K. A. Korobkov \\ Moscow Aviation Institute (National Research University) \\ Volokolamskoe Road, 4, Moscow, A-80, GPS-3, 125993, Russia \\ E-mail: vbusurin@mai.ru, integratedchip.88@gmail.com
}

Received: July 14, 2019

\begin{abstract}
This article presents an experimental investigation of the characteristics of the module based on the optical tunneling effect, which provides information on the nano displacements resonator of the angular velocity transducer. The analysis of the sensitivity of the module based on the optical tunneling effect with increasing amplitude of forced displacements excited by a piezoelectric module is produced. The principle of operation of the module based on the optical tunneling effect, which is based on the dependence of the reflection coefficient of the radiation source structure "medium-gapmedium" on the size of the gap, is determined. The transfer function of the piezoelectric transducer based on the optical tunneling effect with the total optical losses is determined. The theoretical investigation of the transfer function based on the tunneling effect using the module "optical prism - medium - surface simulator of the edge of the ring resonator" is carried out. The results of the experimental investigation are confirmed that when the amplitudes of the input voltages increase, asymmetric amplitudes of the positive and negative half-waves of the output voltages of the module based on the optical tunneling effect are formed. The scheme of the experimental investigation of the transfer function of the module based on the optical tunneling effect, providing the implementation of optical information retrieval in the measurement of angular velocity, is described. The results of the experimental investigation of the optical information sensing unit at different signals of the wave generator show a good agreement with the theoretical model of the module based on the optical tunneling effect and a small change in sensitivity with increasing the angular velocity and amplitude of the displacement of the resonator edge simulator, which should be compensated for the formation of the output signal.
\end{abstract}

Keywords: transducer, angular velocity, optical tunnel effect, piezoelectric transducer, experimental investigation

\section{INTRODUCTION}

The principle of operation of the module based on the optical tunneling effect (MOTE) is based on the dependence of the reflection coefficient of the radiation source for the structure

(C) V. I. Busurin, A. V. Kazaryan, Yin Naing Win, K. A. Korobkov, 2019 
"medium-gap-medium" of the gap. In MOTE, optical radiation passes through the optical prism interacting with the ring resonator (RR) and reaches the photodetector [1-4]. The angle of incidence of the radiation source on the interface between the prism and the gap is chosen such that at a large gap, compared with the wavelength of optical radiation, there is a total internal reflection (TIR). If the size of the gap is comparable with the wavelength of light, then part of the radiation passes (tunnels) through the gap into the second medium and the reflection coefficient of the structure "medium-gap-medium" decreases. Thus, the power of optical radiation reflected from the structure "medium-gap-medium" carries information about the magnitude of the change in the gap and the measured angular velocity[5-10].

When developing an angular velocity transducer, it is necessary to have an adequate description of the real transfer function of the module based on the optical tunnel effect (MOTE), depending on the size of the nano-displacements of the resonator when the measured angular velocity is applied. The validity of the obtained characteristics of the transducer should be based on the results of a comparison of theoretical calculations and experimental studies.

\section{INVESTIGATION OF THE CHARACTERISTICS OF THE MODULE BASED ON THE OPTICAL TUNNELING EFFECT USING THE PIEZOELECTRIC TRANSDUCER}

To check the transfer function of the MOTE of the angular velocity transducer, an experimental investigation was conducted using a piezoelectric transducer based on OTE[11]. For the experimental investigation of the transfer function of MOTE, the scheme is developed that consists of prism of quartz glass 1 , collimating device 2 , fiber optic cable 3 , wave generator 4 , laser module (KIWI-4100) 5, simulator of the edge of the ring resonator 6, piezoelectric transducer 7, photodiode 8, current to voltage Converter 9, digital oscilloscope 10, computer 11 (Fig. 1).

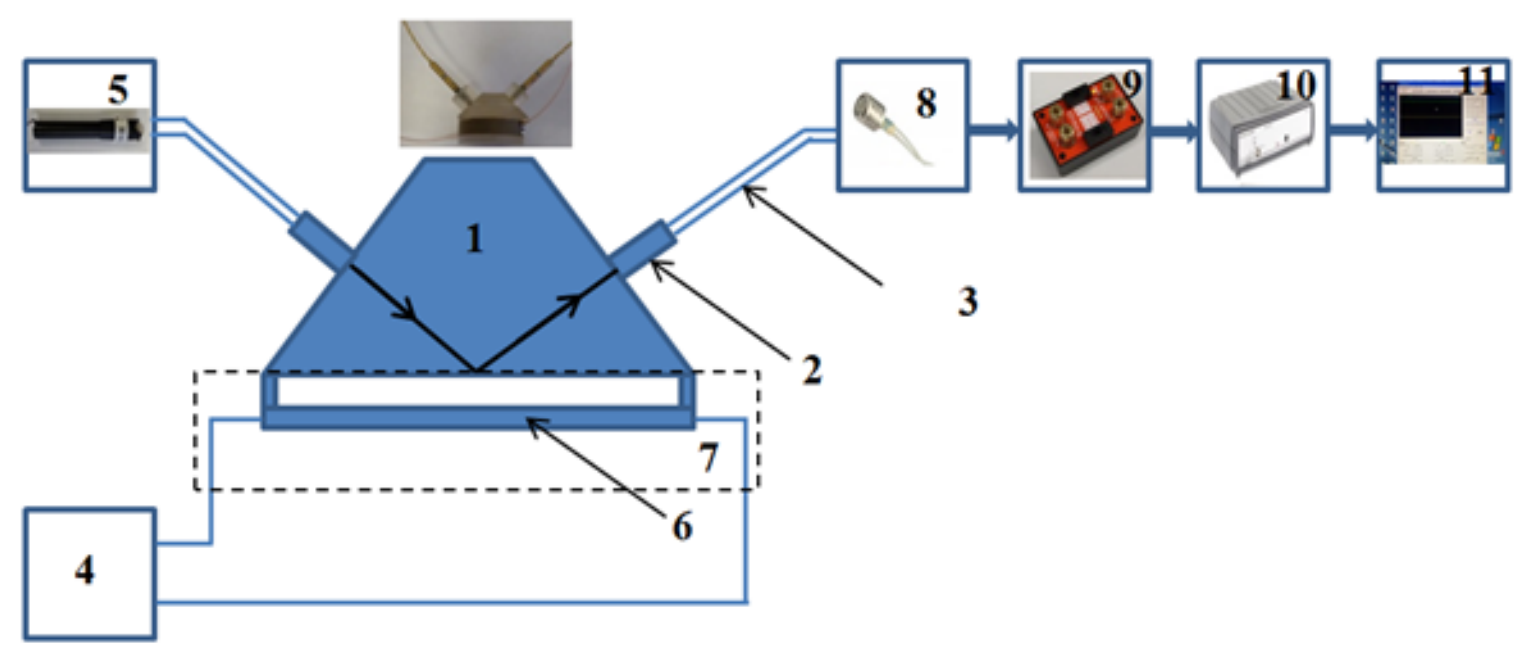

Figure 1. Schematic diagram of experimental setup for the investigation of characteristics for MOTE using piezoelectric transducer

In the mode of generating radiation a laser module (KIWI-4100) was used as a radiation source with a wavelength $\lambda=0.65 \mu \mathrm{m}$ while maintaining a constant power of optical radiation. Periodic changes in the gap were made using excitation of a piezoelectric transducer with different amplitude of the alternating voltage. When applying the exciting voltage to the 
piezoelectric transducer, occurs changing of the working gap $d$ between the surface of the simulator of a part of the edge of the ring resonator and the base of the prism. From the radiation source, the optical signal passes the structure "prism-medium-simulator of a part of the edge of a ring resonator" through an optical fiber. Under the action of the voltage of the wave generator, the magnitude of the nano-displacement of the piezoelectric transducer changes: $y=k_{\mathrm{PT}} \cdot U_{\mathrm{WG}}$, where $k_{\mathrm{PT}}$ - coefficient of piezoelectric transducer, $U_{\mathrm{WG}}-$ voltage of wave generator [12-17].

The size of the working gap $d$ between the surface of the piezoelectric transducer and the base of the prism is determined by the initial gap $d_{0}$ and nano-displacement of the piezoelectric transducer $y\left(U_{\mathrm{WG}}\right)$ :

$$
d\left(U_{\mathrm{WG}}\right)=d_{0}-y\left(U_{\mathrm{WG}}\right) .
$$

When reducing the working gap $d$ due to the approach of the simulator of the edge of the piezoelectric transducer, there is a decrease in the power of the optical signal fed to the photodetector. In this case, the transfer function of such a converter based on OTE, taking into account the total optical loss, is determined by the following formula[18-20].:

$$
U_{\mathrm{I}-\mathrm{U}}\left(U_{\mathrm{WG}}\right)=K_{\mathrm{I}-\mathrm{U}} \cdot S_{\mathrm{PD}} \cdot P_{\mathrm{RS}} \cdot K_{\mathrm{OL}} \cdot R\left[d\left(U_{\mathrm{WG}}\right)\right] \text {, }
$$

where $K_{\mathrm{OL}}$ - coefficient of total optical loss, $K_{\mathrm{I}-\mathrm{U}}$ - coefficient of current to voltage converter, $S_{\mathrm{PD}}$ - sensitivity of the photodiode, $P_{\mathrm{RS}}$ - power of the radiation source, $R\left[d\left(U_{\mathrm{WG}}\right)\right]$ - the reflectivity of the structure "prism-medium-simulator of a part of the edge of a ring resonator".

A theoretical investigation of the transfer function of the module "prism-mediumsimulator of a part of the edge of a ring resonator" was performed with the following parameters: incidence angle of radiation $\theta=47^{\circ}$, wavelength $\lambda=0.65 \mu \mathrm{m}$, power of radiation source $\mathrm{P}_{\mathrm{RS}}=20 \mu \mathrm{W}$, refractive indices of prism and simulator of the interacting part of the resonator $\mathrm{n} 1=\mathrm{n} 3=1.544$, respectively (Fig. 2).

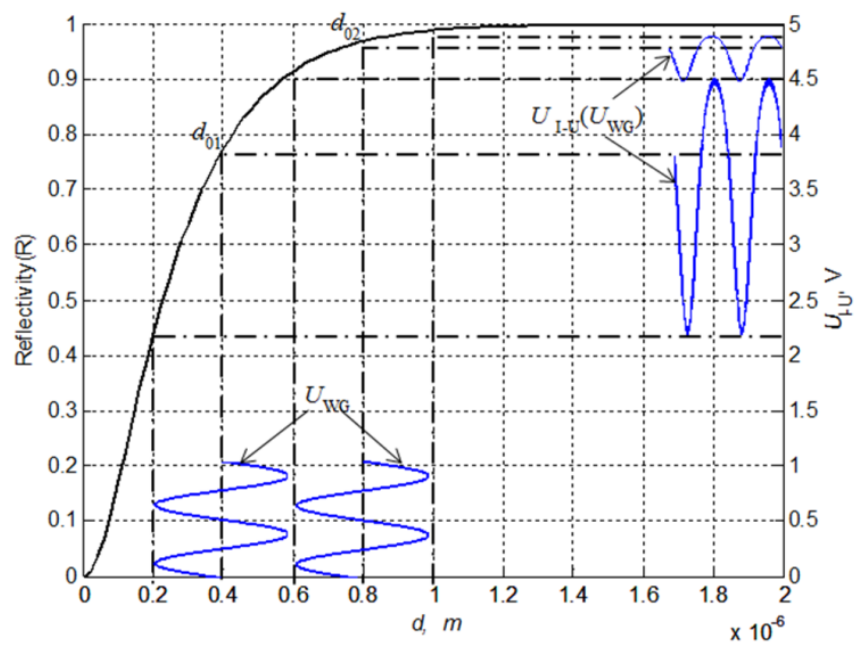

Figure 2. Transfer function of MOTE with variation of the initial gap

It is shown that when the initial gap deviates from the value corresponding to the middle of the quasilinear section, reflectivity of the MOTE changes and the sensitivity of the module "prism-medium-simulator of a part of the edge of a ring resonator" decreases, leading to a decrease in output voltage amplitudes $U_{\mathrm{I}-\mathrm{U}}\left(U_{\mathrm{WG}}\right)$. An increase in the amplitudes of the 
V. I. Busurin, A. V. Kazaryan, Yin Naing Win, K. A. Korobkov

"Experimental investigation of the characteristics of the information sensing module based on the optical tunneling effect of the angular velocity transducer by using a piezoelectric module"

oscillations of the gap $d_{\mathrm{m}}$ ( $\left.\mathrm{U}_{\mathrm{WG}} \mathrm{m}\right)$, proportional to the angular velocity and created by the imitating voltage $\mathrm{U}_{\mathrm{WG}}$ supplied to the piezoelectric transducer, leads to the formation of an asymmetrical output voltage with different amplitudes of positive and negative half waves.

The experimental investigation of the transfer function of the piezoelectric transducer was carried out at various voltage of the wave generator at a frequency of $f=1 \mathrm{kHz}$ (Fig. 3).

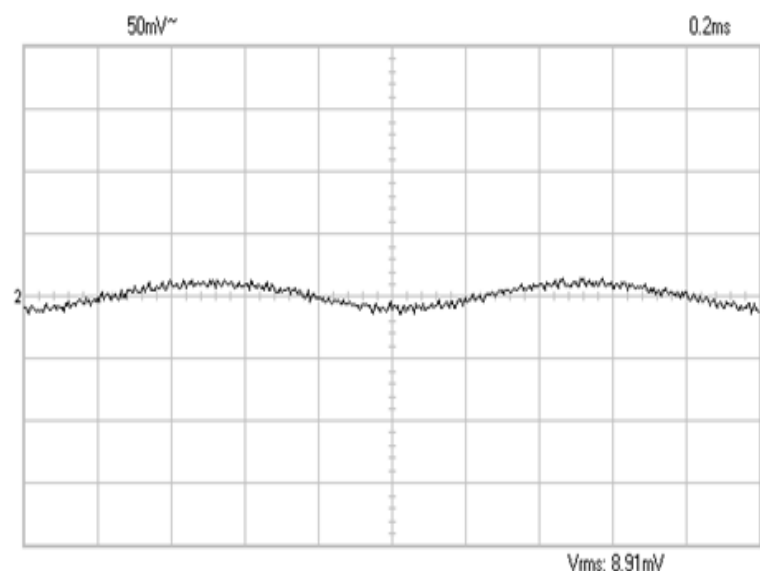

(a)

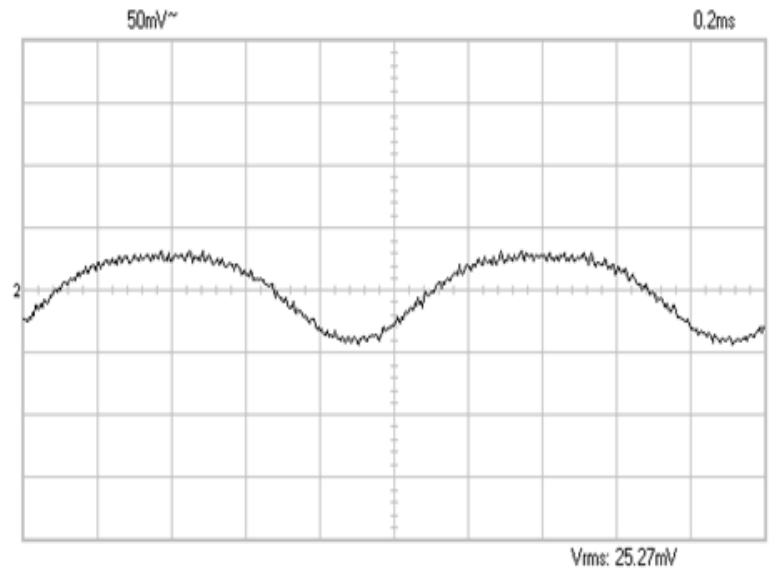

(c)

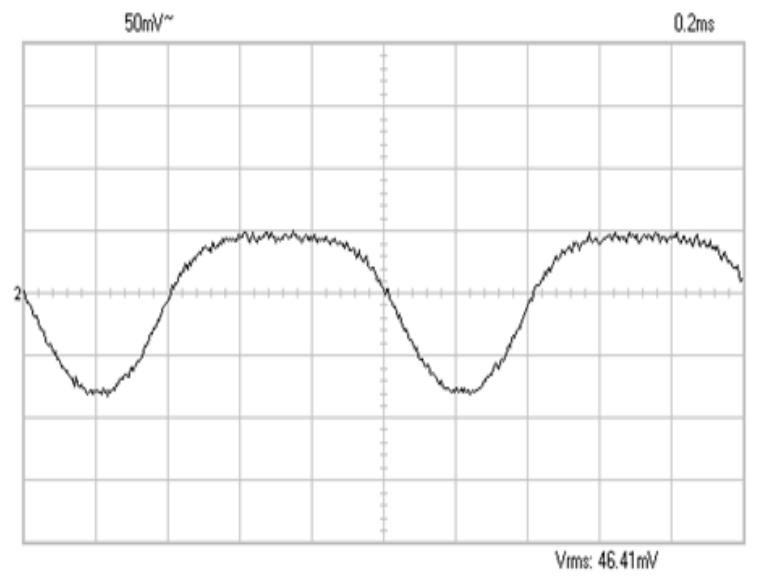

(e)

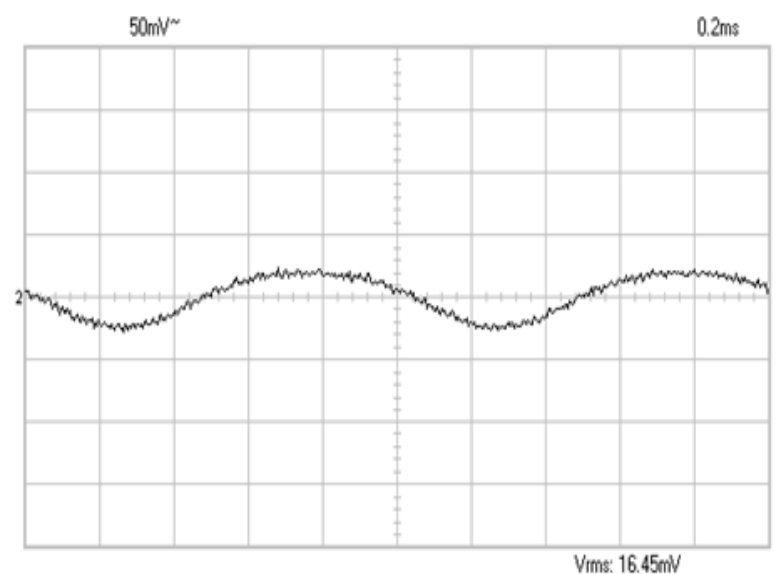

(b)

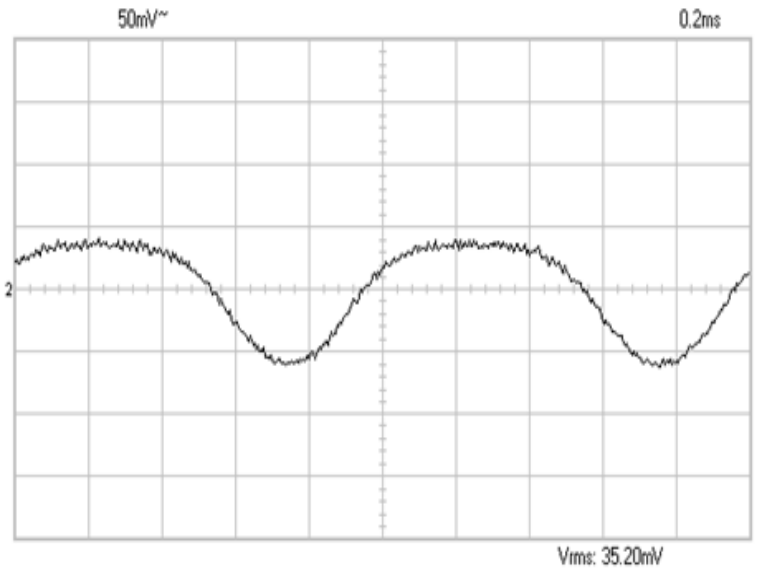

(d)

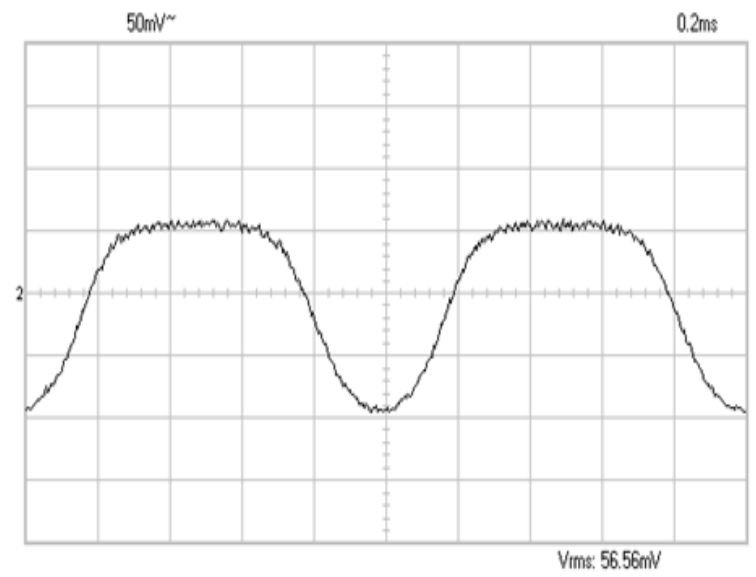

(f)

Figure 3. The relationship of the output voltage of MOTE at a frequency $f=1 \mathrm{kHz}$ on various input voltages: $U_{\mathrm{WG} \mathrm{m}}=20 \mathrm{~V}$ (a); $U_{\mathrm{WG} \mathrm{m}}=40 \mathrm{~V}$ (b); $U_{\mathrm{WG} \mathrm{m}}=60 \mathrm{~V}$ (c); $U_{\mathrm{WG} \mathrm{m}}=80 \mathrm{~V}$ (d); $U_{\mathrm{WG} \mathrm{m}}=100 \mathrm{~V}(\mathrm{e}) ; U_{\mathrm{WG} \mathrm{m}}=$ $=120 \mathrm{~V}(\mathrm{f})$ 
It was confirmed that as the amplitudes of the input voltages increase, asymmetric amplitudes of the positive and negative half-wavelengths of the MOTE output voltages are formed (see Fig. 3), having a difference of up to $38 \%$ according to the results of the experiment (Fig. 4, a). This leads to a decrease in the linearity of the transfer function when simulating the measurement of angular velocity, which requires the introduction of compensating effects. The sensitivity of MOTE to periodic changes in the gap remains approximately the same due to the compensation of the decrease in sensitivity for a positive half-wave with its increase for a negative half-wave.

A comparison was made of experimental and theoretical data on the transfer function of the piezoelectric transducer for various signals of wave generator and initial gaps (Fig. 4, b).

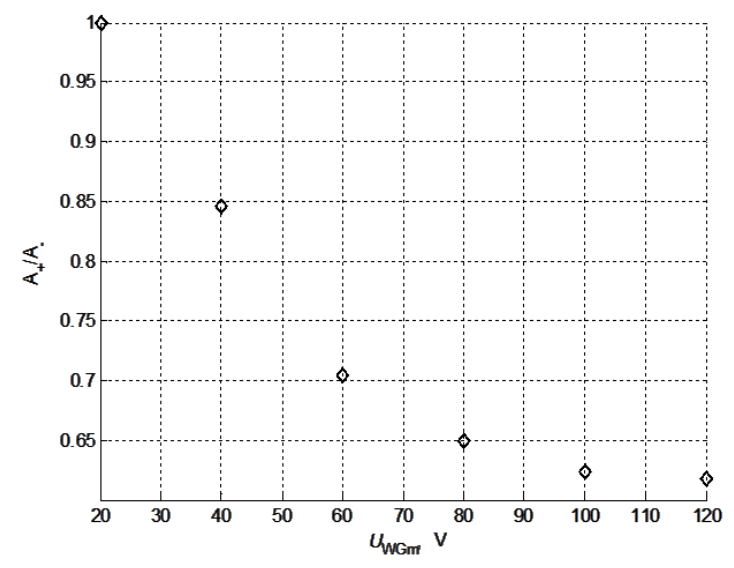

(a)

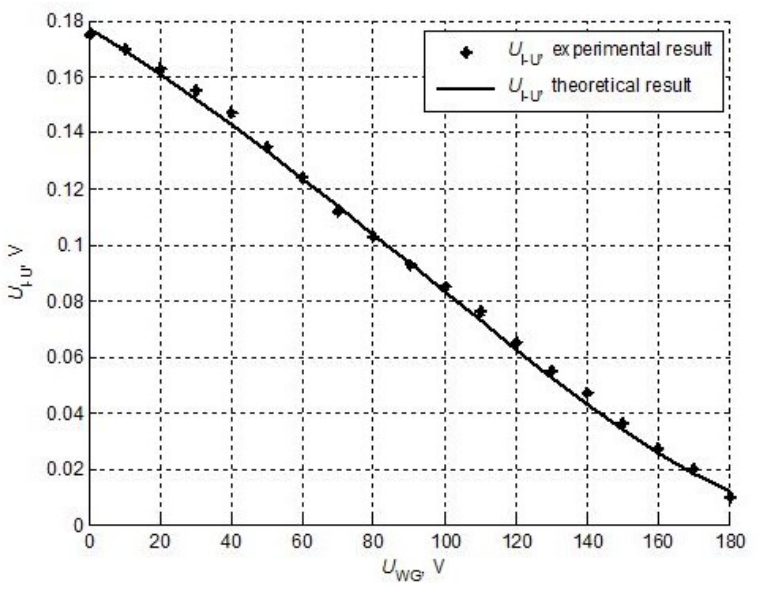

(b)

Figure 4. The ratio of the amplitude of the positive and negative half-waves $U_{\mathrm{WG}}(a)$; comparison of theoretical calculations and experimental investigation of the information retrieval module based on the optical tunneling effect $(b)$

A comparison of theoretical calculations and experimental studies shows that the use of theoretical dependences in the calculation of transducers based on the optical tunnel effect gives good agreement with the experimental data.

\section{CONCLUSION}

A theoretical investigation of the transfer function based on the optical tunneling effect using the module "prism - medium - surface of the simulator of the edge of the ring resonator" is described. A scheme for the experimental investigation of the MOTE transformation function is described, which provides the realization of optical information retrieval module in measuring the angular velocity. The results of an experimental investigation of an optical information pickup site with various wave generator signals show good agreement with the theoretical model of MOTE and a small change in sensitivity with increasing angular velocity and amplitude of displacements of the resonator edge simulator, which should be compensated for when forming the output signal.

This work was supported by the Russian Foundation for Basic Research (Grant No. 19-08-00108). 


\section{REFERENCES}

1. Udd, E., \& Spillman, W. B. Jr. (Eds.) (2011). Fiber optic sensors: An introduction for engineers and scientists ( $2^{\text {nd }}$ ed.). New Jersey : John Wiley \& Sons, Incp.

2. Xia, D., Yu, C., \& Kong, L. (2014). The development of micromachined gyroscope structure and circuitry technology. Sensors, 14, 1394-1473. doi: 10.3390/s140101394.

3. Gallacher, B. J., Burdess, J. S., \& Harris, A. J.(2001) Principles of a three-axis vibrating gyroscope. IEEE Transactions on Aerospace and Electronic Systems, 37, 1333-1343. doi: 10.1109/7.976969.

4. Busurin, V. I., Korobkov, V. V., \& Win, Yin Naing. (2017). Issledovaniye vliyaniya parametrov kol'tsevogo rezonatora na kharakteristiki tryokhosevogo optoelektronnogo preobrazovatelya uglovoy skorosti [Investigation of the ring resonator parameters influence on the characteristics of the three-axis optoelectronic angular velocity transducer]. Doklady TUSUR [Proceedings of TUSUR], 20(4), 43-49. doi: 10.21293/1818-0442-2017-20-4-43-49. (in Russian).

5. Dennis, D. (Ed.). (1998). Fiber optic test and measurement. Prentice-Hall PRT.

6. Fraden, J. (2010). Handbook of modern sensors: Physics, designs and applications (4 ${ }^{\text {th }}$ ed.). New York: Springer-Verlag. doi: 10.1007/978-1-4419-6466-3.

7. Nash, W. A., \& Potter, M. C. (2011). Schaum's outline of strength of materials (5th ed.). New York : The McGraw-Hill Companies, Inc.

8. Kostsov, E. G. (2009). Status and prospects of micro- and nanoelectromechanics. Optoelectronics, Instrumentation and Data Processing, 45, 189-226. doi: 10.3103/S8756699009030017.

9. Busurin, V. I., Win, Yin Naing, \& Zheglov, M. A. (2019). Effect of linear acceleration on the characteristics of an optoelectronic ring transducer of angular velocity and its compensation. Optoelectronics, Instrumentation and Data Processing, 55, 309-316. doi: 10.3103/S8756699019030142.

10. Evstifeev, M. I., Kovalev, A. S., \& Eliseev, D. P. (2014). Electromechanical model of RR type MEMS gyro with consideration for the platform vibrations. Gyroscopy and Navigation, 5, 174-180. doi: $10.1134 / \mathrm{S} 2075108714030043$.

11. He C., Zhao Q., Huang Q., Liu D., Yang Z., Zhang D., \& Yan G. (2015).A MEMS vibratory gyroscope with real-time mode-matching and robust control for the sense mode. IEEE Sens. J. 15:2069-2077. doi: 10.1109/JSEN.2014.2371456.

12. Cheng Li, Bo Yang, Xin Guo, \& Lei Wu.(2019). A Digital Calibration Technique of MEMS Gyroscope for Closed-Loop Mode-Matching Control. Micromachines (Basel). 2019 Jul 25;10(8). pii: E496. doi: 10.3390/mi10080496.

13. Busurin, V. I., Korobkov, V. V., Lwin, Naing Htoo, \& Tuan, Phan Anh. (2016). Static and dynamic characteristics of angular velocity and acceleration transducers based on optical tunneling effect. Journal of Physics: Conference Series, 737, 012045. doi: 10.1088/1742-6596/737/1/012045.

14. Ovchinnikova, N., Panferov, A., Ponomarev, V., \& Severov, L. (2014). Control of vibrations in a micromechanical gyroscope using inertia properties of standing elastic waves. In: Preprints of the 19th World Congress "The International Federation of Automatic Control” (pp. 2679-2684). Cape Town, South Africa: IFAC.

15. Busurin, V. I., Gorshkov, B. G., Gorshkov, G. B., Grinshtein, M. L., \& Taranov, M. A. (2017). A factor limiting the accuracy of optical loss measurements in single-mode fibres: 'frozen-in' inhomogeneities of the Rayleigh backscatter coefficient. Quantum Electronics, 47, 83-86. doi: 10.1070/QEL16201

16. Evstifeev M.I., \& Eliseev D.P. (2016). Mathematical model of RR-type micromechanical gyro capacitive comb-type sensors with account for vibrations. Scientific and Technical Journal of Information Technologies, Mechanics and Optics, Vol. 16, No. 3, pp. 541-549.

17. Evstifeev M.I., Eliseev D.P., \& Chelpanov I.B. (2015). Enhancing the mechanical resistance of micromechanical gyros. Gyroscopy and Navigation, Vol. 6, No. 2, pp. 115-122. doi: 10.1134/S2075108715020042

18. Geen J. (2004). Progress in integrated gyroscopes. IEEE Aerospace and Electronic Systems Magazine, Vol. 19, No. 11, pp. 12-17. doi: 10.1109/MAES.2004.1365660 
"Instrumentation Engineering, Electronics and Telecommunications - 2019"

Proceedings of the V International Forum (Izhevsk, Russia, November 20-22, 2019)

19. Busurin, V. I., \& Tuan, P. A. (2016). Micro-opto-electro-mechanical angular velocity transducer based on the optical tunneling effect. Optoelectronics, Instrumentation and Data Processing, 52, 210-215. doi: $10.3103 / \mathrm{S} 8756699016020151$.

20. Busurin, V. I., Korobkov, V. V., \& Yin Naing Win.(2016). Investigation of Characteristics of the Optoelectronic Ring Wave Angular Velocity Transducer. Merhatronika, Avtomatizatsiya, Upravlenie, Vol.17, no 5, pp. 340-346. doi: 10.17587/mau/16.340-346. 